\title{
Using a consistency factor for detection and attribution of anthropogenic impacts on phenological phases in Germany
}

\author{
Sebastian Lehner ${ }^{\mathrm{a}, \mathrm{b}, *}$, Christoph Matulla $^{\mathrm{b}}$, Helfried Scheifinger $^{\mathrm{c}}$ \\ ${ }^{a}$ Department of Meteorology and Geophysics, University of Vienna, Vienna, Austria \\ ${ }^{b}$ Climate Impact Team (CIT), Zentralanstalt für Meteorologie und Geodynamik (ZAMG), \\ Vienna, Austria \\ ${ }^{c}$ Customer service Section Climate, Zentralanstalt für Meteorologie und Geodynamik \\ $(Z A M G)$, Vienna, Austria
}

Keywords:

detection, attribution, climate change, anthropogenic impact, phenology

Preprint submitted to EarthArXiv (non-peer reviewed version). Original manuscript submitted to International Journal of Climatology.

\footnotetext{
${ }^{*}$ Corresponding author

Email address: sebastian.lehner@zamg.ac.at (Sebastian Lehner)
} 


\section{Abstract}

2

3 cycle of vegetation flora and fauna. Although it is generally understood that

4 anthropogenic mechanisms play a major role in the warming trend of the

5 climate and that the timing of such phases, especially spring timing events,

6 depends largely on the temperature, the link has yet to be quantitatively

7 shown for different kind of areas on a regional scale, due to high intrinsic

8 noise.

9 In this study, an end-to-end analysis to external forcings was carried out,

linking the earlier timing of phenological spring timing events to changed climate conditions (increasing temperature) and this change in the environment to anthropogenic forcing, for the region of interest: Germany.

Besides a large ensemble originating from six different General Circulation Models (GCMs), driven with various kinds of forcings, the E-OBS data set was used as observational reference for near-surface air temperature and the PEP725 for phenological observations. The latter contains over 100 different phenological phases, from which - after quality checking - 12 phases are evaluated.

To overcome the scale differences, a quantile mapping bias correction approach was used to downscale the GCM data. The generation of simulated phenological time series was done with a temperature-day-sum model, which had to be calibrated and optimised beforehand.

The signal-to-noise ratio was increased by generating samples of 50-year running trends, which make up the basis for the statistical evaluation. U-values from the Mann-Whitney U-Test represent the foundation, on which the null 
hypothesis for detection, that the observed changes can be explained with naturally forced climate conditions only, was tested. Based thereupon, a newly defined consistency factor was constructed, which allowed the assessment of anthropogenic impact on phenological phases. Observed changes in phenological phases were successfully attributed to anthropogenic impacts.

\section{Introduction}

Detection is the process of showing that an observed change in some variable is significantly different from what is expected, if only internal climate variability or naturally forced climate states are considered. Formulated in a statistical sense, this boils down to test if the null hypotheses, that observed changes can be explained by internal variability or naturally forced conditions alone, has to be rejected. In the attribution part, it has to be shown that this discrepancy - if found - is consistent with a combination of other, differing kind of forcings and that it is inconsistent with remaining physically plausible explanations, which exclude the forcings investigated before (see e.g. Hegerl et al. (2010); IPCC-AR5 (2014)).

There has already been quite some work done in assessing the fingerprint of human activity on different elements of the biosphere and on more general detection and attribution tasks covering all aspects of the climate system. Related to meteorological variables, such as temperature, precipitation or circulation systems, especially the focus on extreme event attribution, assessing the change in likelihood and/or magnitude due to anthropogenic impacts, has gained a lot of a traction (see e.g. Walther et al. (2002); Ahas et al. (2002); Root et al. (2003), Menzel et al. (2006); Rosenzweig et al. (2008) for the for- 
mer, or Hegerl et al. (1997); Karoly et al. (2003); Stone et al. (2009),Dean \& Stott (2009) Rosenzweig \& Neofotis (2013); Stott et al. (2016) for the latter). We considered here the task of investigating long-term shifts in phenological timing events, hence extreme detection and attribution techniques are not useful for our goal.

The number of detection and attribution studies has been increasing during the last 20 years. This is largely due to improvements of General Circulation Models (GCMs), as well as the fact, that with time, a potential human influence on the climate is more likely to be observed and finally, that especially in recent years, public interest in attribution questions have increased significantly. In general, the smaller the region, the more difficult the detection as well as the attribution. The reason therefor being, that random noise components represent a relatively larger part of an observed signal, or in other words, variability of climate variables is inversely proportional to the size of their respective region they represent. To overcome this issue, the signal-to-noise ratio has to be increased (Hegerl et al. (2010); IPCC-AR5 (2014)). In this study, the goal was to circumvent this issue with the use of long-term trends (see e.g. Scheifinger et al. (2003)).

Combining detection and attribution is a natural step in analysing changing patterns of observed plant species developments. Phenological station records were investigated and tested, if the observed change in entry dates can be explained by internal climate variability and/or naturally forced climate conditions alone. Twelve different phenological phases were inspected, which range from early spring to late summer timing, thus covering a large part of the vegetation cycle. 
The analysis is considered an end-to-end analysis based on Stone et al. (2009). This implies differently forced climate model output variables are used and fed in an ecological model, from which the output is evaluated. To capture all needed information, an ensemble consisting of multi-model 'pi-Control', 'historical' and 'historicalNat' GCM experiments was needed. On the other end of the spectrum, a phenological temperature-day-sum model was used to generate simulated entry dates from temperature series (see Hunter \& Lechowicz (1992), Chuine et al. (1998, 1999, 2000) or for a more general overview in plant development models Chuine et al. (2003)). In general, before temperature data from the GCMs can be used on a regional scale, the data has to be downscaled. Variables in the GCM domain represent the large-scale state, but to be able to compare them to E-OBS data and the phenological observations, they must be transferred to the local-scale. This was done via a quantile mapping bias correction approach, applied on a daily basis, where the E-OBS observational data was used as reference, or 'true state' (see Panofsky et al. (1958), Maurer (2010), Abatzoglou \& Brown (2012), Thrasher et al. (2012), Maurer et al. (2013)). Important aspects regarding the use of models and their associated uncertainties are discussed in Hegerl \& Zwiers (2011). The resulting entry dates time series from the phenological model were then subject to the calculation of running 50-year trends. Furthermore, the Mann-Whitney U-Test was used as statistic measurement tool to assess the null hypothesis (Mann \& Whitney (1947); Wilcoxon $(1947,1992))$. It is a non-parametric statistical test about homogeneity, which checks the significance whether two distributions originate from the same basic population. Here, it was adapted for the detection 
of a discrepancy between observations and internal or naturally forced climate conditions, which can be assessed using the significance of the resulting $U$-values from two tested samples. Additionally, the $U$-values were then used to define a, so called, consistency factor, which takes into account two signals and compares the overlap/distinctness of those two to a third signal (e.g. observations). The constructed consistency factor represents how consistent the third signal is with the distinctness of the former two. Using naturally forced data as one sample and the combined anthropogenic \& naturally forced as second, the distinctness between those two represents the anthropogenic impact (neglecting physically possible interactions). Hence, the resulting factor for consistency validates if the observations are consistent when anthropogenic forcing is included (consistency hypothesis). The same procedure was applied to naturally forced and 'piControl' experiments, to evaluate the impact of natural forcing.

Used data is presented in section 2 and the adapted methods in section 3. The quantile mapping bias correction approach is explained in section 4 and section 5 is devoted to the optimisation, calibration and validation process of the phenological model. The detection analysis is shown and discussed in section 6 and the attribution issue in section 7. Finally, the paper ends with concluding remarks containing an outlook.

\section{Data}

A range of different data was needed to fulfill the set out tasks. For phenological data, the PEP725 data set was used (Templ et al., 2018). As observational reference, the gridded E-OBS data was employed (Haylock et al., 
2008; Hofstra et al., 2008; Van den Besselaar et al., 2011). Large-scale information was utilised from six different GCM models, each providing a small ensemble on naturally, internally and anthropogenically \& naturally (combined) forced conditions (Taylor et al., 2012).

\subsection{Phenological Station Observations}

The PEP725 data set (Templ et al., 2018) is a large, to some extent quality-controlled, collection of phenological station data for Europe. It consists of more than 100 different phenological phases, more than 20000 registered stations (in the form of latitude, longitude, altitude) and dates back into the 19th century. For this study, because of availability and quality reasons, only data from 1951 onward is exploited.

An example for the general spatial distribution can be seen in figure 1. For the most part, this (stationary image due to averaging) mirrors the overall distribution in space and time quite accurately. Although not explicitly shown, the latter is true, due to a large number of observations being present each year (the United Kingdom being the only exception, where data was observed only in the last couple of decades). The recorded quantity has been decreasing over the last decades, however, this decline is not of significance, relative to the overall amount of information available and much more importantly, this reduction has no spatial component and happens everywhere to approximately the same extent.

First, all of the available phenological data was sieved by a few characteristics, namely:

(i) spatial homogeneity over time, 
(ii) enough observations per year to guarantee statistical robustness,

(iii) phenological observations which are mainly responsive to temperature. While (i) \& (ii) act as general restrictions on the availability of the data and are quite straightforward, the last point has to be explicitly checked for. The reason for (iii) as criterion is simply a consequence of the temperature-daysum model. Phases with complex interactions between other meteorological or non-meteorological factors (often autumn phases) cannot be modeled as well with such a model, as those which are mainly responsive to temperature (for responsiveness of ecological systems see e.g. Walther et al. (2002); Menzel et al. (2006)). Running 30-year trends of entry dates in compliance with temperature trends for the same region serve as measure. If the third quartile of those trends is negative, the phase is determined to be responsive enough (not shown explicitly here). The twelve phases leftover are listed in table 1. To guarantee statistical robustness, only Germany provided a large enough record in time and space for these phenological phenomena. The earliest long term mean entry date is 110.53 for Taraxacum officinale - beginning of flowering and the latest 244.58 for Sambucus - first ripe fruits, hence, a major part of the vegetation period from early spring to late summer is covered. However, note that they are not equally distributed. Most of the phases occur before the summer solstice. The observed change in distribution from 1951-1970 to 1996-2015 is shown in figure 2.

\subsection{E-OBS - Observational data}

Near-surface air temperature from the E-OBS data set (v17.0) provides gridded, observational, 'local-scale' information on a daily basis. It is available on a 0.25 degree regular lat-lon grid and covers $25 \mathrm{~N}-75 \mathrm{~N}$ x $50 \mathrm{~W}-75 \mathrm{E}$, 
extending from 1950 onward. Average temperature as well as altitude information for each grid-point was used. The data set contains only valid values over land. For more information on the data itself, see e.g. Haylock et al. (2008); Hofstra et al. (2008); Van den Besselaar et al. (2011). The spatial range was dependent on the GCMs and is listed below.

The temperature data serves as a reference for bias correcting the GCMs, as well as for the optimisation process, which is needed to derive the phenological model parameters. Furthermore, the modeled phenological entry dates - driven with E-OBS data - represent the observational state onto which the GCM data is evaluated against in the statistical analysis. The altitude information is used for a multiple linear regression model relating latitude, longitude and altitude to phenological entry dates and is explained in more detail in section 3 .

\subsection{General Circulation Models - CMIP5}

A range of different models and experiments from the CMIP5 (Coupled Model Intercomparison Project Phase 5, see Taylor et al. (2012)) was used, delivering the large-scale information for near-surface air temperature. To be able to assess different impacts on phenological entry dates, data sets with distinct underlying forcings were needed. The 'piControl' (pre-industrial Control) experiment is the foundation of every GCM and was used to determine internal climate variability. To be able to evaluate the impact from natural forcing, (e.g. volcanic activity, solar variability) 'historicalNat' experiment runs were used and compared with 'historical' ones, which are additionally driven by anthropogenic factors (e.g. greenhouse gases).

A short overview of all available GCMs is shown in table 2. The differing 
spatial resolution between the models has to be taken into account. Furthermore, according to e.g. Von Storch et al. (1993), Tett et al. (1999), Stott et al. (2000), a certain minimum threshold of grid-points per GCM should be considered. Approximately 8 x 8 grid boxes are the minimum quantity, in order to capture the necessary state of a system condition adequately by a GCM in general. A bounding box with extents $[0,20]^{\circ} \mathrm{E} \&[42,58]^{\circ} \mathrm{N}$, is used to spatially average the data, which is deduced from the compromise that enough GCM grid-points are covered, while being as close as possible to the spatial extent posed by the phenological station observations.

More general information on GCMs and their characteristics can be found in the Taylor et al. (2012) \& IPCC-AR5 (2014) and for specific models in Bentsen et al. (2013) \& Voldoire et al. (2013) - NorESM1-M \& CNRM-CM5 respectively.

\section{Methods}

\subsection{Quantile mapping bias correction}

For the consistency analysis, all of the data was spatially averaged, to generate representative time series for Germany. Therefore, the different data sets had to be made comparable to each other, so that they conform to a matching statistic (GCM - large-scale, E-OBS - local-scale). This downscaling process was done through a quantile mapping bias correction. For this application on temperature data, this method retains sufficient skill, hence there is no need in heading for more sophisticated downscaling techniques (which would probably be required when working with e.g. precipitation data (see e.g. Thrasher et al. (2012)). 
The GCM and E-OBS data sets were detrended in a two-way fashion, with the first period being 1950-1985 and the second 1986 onward until the end of the respective available time range (shifting means were accounted for), to remove instationarities (the observed, non-linear trend characteristic). The bias correction follows the general approach in e.g. Thrasher et al. (2012) and was done on a daily basis. For every yearday, the distribution of mean daily temperature from a model was modified such that it matches that of the observations. Therefore, the model as well as the observational data were sorted and the corresponding empirical cumulative distribution functions (ecdf) calculated. The resulting ecdf for the GCM data, was then interpolated in a piecewise linear fashion to the GCM model. In the next step the observed ecdf (from the E-OBS data) was inverted. This was done via reversing $x$ and $y$ in the linear interpolation, hence determining the interpolants for the bins located at the observational ecdf and evaluated at the GCM model ecdf values. The resulting ecdf is then the corrected distribution for the GCM model values, which have to be sorted back to the original time of each point.

\subsection{Phenological station data to E-OBS grid}

First, the phenological station data had to be brought to the E-OBS grid. Therefor, a multiple linear regression model (depending on latitude, longitude and altitude) was derived. For every E-OBS grid-point in Germany, all observations within a $100 \mathrm{~km}$ radius were either averaged - if the altitude difference of the phenological stations was less than $100 \mathrm{~m}$ to the corresponding grid-point, or, if the altitude difference was more than $100 \mathrm{~m}$, corrected with the altitude coefficient derived from the MLR model. Latitude and longitude do not need to be corrected in the same fashion, because they exhibit no sig- 
nificant dependency to the entry date, for the region of interest. Quantiled entry dates in relation to binned latitude, longitude and altitude ranges are shown in figure 3 . The variability of the entry dates along latitude and longitude can be explained when taking into account the average topography in each respective bin (not shown). Hence, for the regional extent discussed here, only the effect of altitude was of significance when aggregating data from different heights. Altitude regression coefficients from the MLR model are shown in table 3 .

\subsection{Phenological Model}

Phenological (especially spring time) phases correlate highly to the temperature development for a given year on a daily basis. A simple temperatureday-sum model according to Chuine et al. (1999) consists of the following parts. The entry date $y$ is determined such that

$$
f_{c}(y)=F^{*}
$$

with

$$
f_{c}(t)=\sum_{t_{0}}^{t} R_{f}\left(x_{t}\right)
$$

where $f_{c}$ is the state of forcing, $F^{*}$ is a critical forcing state value describing the transition to the phase occurrence, $R_{f}\left(x_{t}\right)$ is the forcing rate function for the average daily temperature $x_{t}$ on day $t$ and $t_{0}$ is the starting date of summation. The forcing rate function (or development rate) is a simple step function of the form

$$
R_{f}\left(x_{t}\right)= \begin{cases}0 & \text { if } x_{t} \leq T_{b} \\ x_{t}-T_{b} & \text { if } x_{t}>T_{b}\end{cases}
$$


with $T_{b}$ the base temperature. Three parameters need to be determined for the model to work properly: the starting day $t_{0}$, the base temperature $T_{b}$ and the critical forcing state value or summation threshold $F^{*}$. Using phenological and temperature observations, the optimal set of parameters can be attained with an optimisation process. A brute force (due to a feasible amount of calculation needed) and a probabilistic algorithm were applied for this task (see Metropolis et al. (1953) for the latter). Due to temperature being the only driving factor, the model is very sensitive to shifts in the input series. Therefore, if using an optimised model for a different range (climatology) of temperature series, the goodness of the results can not be guaranteed. Consequently, it is important that temperature series using the same set of parameters should exhibit statistics comparable to the reference series from which the optimal parameters were derived. Note that, although the model itself might be quite simple, it nevertheless works very well for temperature-responsive phases, which are - by design - investigated here.

\subsection{Detection and attribution technique}

The Mann-Whitney U test is a non parametric statistical test, which examines homogeneity between two samples (Mann \& Whitney (1947); Wilcoxon $(1947,1992))$. A few assumptions are implied: (i) the two samples are independent of each other, (ii) the data corresponds to some ordinal scale i.e. a rank can be associated to each sample, to determine which is greater in a statistical sense, (iii) the null hypothesis $H_{0}$ for which the test is done, suggests that both signals originate from the same basic population and (iv) the alternative hypothesis $H_{1}$ leads to the original distributions being not from the same basic population. All of these assumption were readily fulfilled. 
Technical details can be found in e.g. Schönwiese (2013).

After ranking the trend values of two data sets ('historical': $H$ and 'historicalNat': $N$ ), which were compared, the corresponding $U$ values were calculated as follows:

$$
U_{X}=R_{X}-\frac{\left(n_{X}\left(n_{X}+1\right)\right)}{2},
$$

where $U_{X}$ is the $U$ value for the trends of the data set $X$ (i.e. either $H$ or $N), R_{X}$ is the rank sum of $X$ and $n_{X}$ is the corresponding sample size.

$$
U_{H N}=\min \left(U_{H}, U_{N}\right)
$$

represents the minimum of both calculated $U$ values and is an indication about the overlap of two distributions. $U_{H N}$ can then be normalised with the total number of samples multiplied with each other:

$$
U_{H N}^{*}=\frac{U_{H N}}{n_{H} n_{N}}
$$

This spans the $U^{*}$ value in a range between 0 and 0.5 . A value of 0 suggest no, 0.5 maximum overlap of the sample distributions. Based thereupon, a 'consistency factor' can be defined:

$$
c=\left(0.5-U_{H N}^{*}\right)+2 U_{H O}^{*}\left(0.5-U_{H N}^{*}\right),
$$

where $U_{H O}^{*}$ corresponds to the $U^{*}$ value derived between the two samples $H$ 'historical' and $O$ observations. The first term on the right-hand side represents the difference between the distributions of the two signals - one being the 'historical', the other the 'historicalNat' trends - in the form of $U_{H N}^{*}$, subtracted from 0.5. Thus, the whole term is large for no overlap between the two samples and small the other way around. In the second term, the 
difference between observations and historically forced runs, $U_{H O}^{*}$, is weighted with the former term, which represents - in this case - the anthropogenic effect (distinctness between 'historical' and 'historicalNat'). Therefore, $c$ represents how consistent the observations are with anthropogenic forcing. The value range for the consistency factor is $[0,1]$ and following, three cases are outlined here:

(i) If the trend distributions of the 'historical' and 'historicalNat' forced runs are different, $U_{H N}^{*}$ will be close 0 , hence the first term close to 0.5. Depending on the similarity of observations and 'historical' distributions, in the optimal case - for matching pdfs - $U_{H O}^{*}$ will be close to 0.5 resulting in the consistency factor being close to 1 ( 1 being the perfect score).

(ii) If $U_{H N}^{*}$ is close to 0.5 , natural forcing prevails and the first as well as the second term will both be close to zero, regardless of the value of $U_{H O}^{*}$. The interpretation in this case is, that the two signals do not allow a distinction between each other, thereby disallowing possible attribution.

(iii) For $U_{H N}^{*}$ and $U_{H O}^{*}$ both being close to 0 , the resulting consistency factor will be around 0.5. Although detection can be successfully reached (if the observations are distinct from the 'historicalNat' and 'piControl' distributions), attribution might not be completely clear. A look at the trend distributions themselves can help deduce a concluding statement. E.g. there could be a case, where the anthropogenic forcing is visible and causes a shift in the direction of the observations, but the amplitude may be too small to be statistically consistent. Furthermore, it is important to not only look at the value of $c$ in a nutshell, but to plot 
it, using the $U^{*}$ values on the $\mathrm{x}$ and $\mathrm{y}$ axis, which will be discussed in section 7 .

Furthermore, adding a naming convention for $c$ in the above case for clarity (equation 7): $c=c_{H N O}$, then $c_{N P O}$ and $c_{H P O}$ (see equation 8 and 9 respectively) can be computed as well, which were used to test the consistency for physically plausible alternatives. This convention reads as follows: $c_{x y z}$ represents the consistency factor for which the distinctness between $x$ and $y$ acts as weighting on the distinctness between $x$ and $z$, thereby assessing the consistency between $x$ and $z$.

To summarise, first of all, a successful detection is achieved, if the $U^{*}$ values between the observations and 'historicalNat' as well as between observations and 'piControl' are not significant based on the Mann-Whitney U Test, indicating they originate from different underlying distributions. Second, the consistency factor allows the assessment which forcing - if any - is consistent with observations, thereby attributing the observed change to a cause. Although as mentioned above, to grasp the full picture, the $c$ values should always be plotted using their $U^{*}$ values, a threshold of $c=0.5$ was derived using synthetic data (not shown), which can be used for a quick evaluation. If $c>0.5$, attribution is suggested.

$$
\begin{aligned}
& c_{N P O}=\left(0.5-U_{N P}^{*}\right)+2 U_{N O}^{*}\left(0.5-U_{N P}^{*}\right), \\
& c_{H P O}=\left(0.5-U_{H P}^{*}\right)+2 U_{H O}^{*}\left(0.5-U_{H P}^{*}\right),
\end{aligned}
$$




\section{Preparing the GCM data}

\subsection{Characteristics of GCMs}

Although GCMs simulate atmospheric and oceanic circulation on the basis of dynamical equations, the coarse resolution prevents the explicit treatment of sub-scale processes, which hence have to be parameterised. This in turn, can lead to errors in modeling processes happening on smaller - not resolved - scales. First of all, the characteristics of the different GCM temperature series are investigated. Reasonable modeling results can only be guaranteed, if the GCM temperature data exhibits no systematic biases to the reference data, from which the phenological model was calibrated (EOBS data).

Inter-year distribution of temperature and especially the development up to the entry-date of a phenological phase has a big impact on the phenological model results. If the temperature in the weeks before an entry-date features a positive Bias, the model prediction will always tend to generate earlier entry-dates, resulting in a negative Bias for simulated phases. Because of this sensitive interaction, the day-to-day distribution of near-surface temperature is of high importance and therefor the bias correction has to be made on a daily basis.

\subsection{Bias corrected GCM data}

10 year averaged mean year E-OBS data with a rolling 45 day filter uncovers the non-linear trend over the period of interest (not shown). Furthermore, before the correction was applied, the time series were made to be stationary. 
To incorporate the former issue into the bias correction, the detrending was split up into two unique periods, the first being 1950-1985 and the second 1986 onward until the end. Note that 'until the end' loosely refers to the end of the congruent period between a single GCM run and the E-OBS data. So e.g., if said run features data until 2005, E-OBS data afterwards was not considered, in order to exclude possible errors arising from detrending based on data which is not included in the Bias correction itself.

Figure 4 shows the Root Mean Square Error (RMSE) for mean near-surface temperature per yearday (over the full time span) for the 'historical' GCM ensemble (blue) and the 'historicalNat' GCM Ensemble (green) with respect to E-OBS data. Dashed lines correspond to raw, solid lines to bias corrected data. The corrected GCM data was then forwarded and fed into the calibrated phenological model.

\section{Generating the simulated phenological entry dates}

\subsection{Optimising the phenological model}

As already mentioned before, three parameters - namely the start day of summation (hereafter $t_{0}$ ), the base temperature (hereafter $T_{b}$ ) and the critical temperature sum (hereafter $F^{*}$ ) - need to be optimised. Due to the nature of optimisation processes, there are many iterative options available to solve this problem. The two realised ways are discussed and one shown (due to both methods generating comparable results). An application of the one not shown - simulated annealing - can also be found in Chuine et al. (1998).

A quantity needs to be defined, in order to assess the quality of an optimisation iteration. The summed squared residual $(S S R)$ day was used therefor, 
which behaves similarly to the root mean square error (RMSE).

A very straightforward optimisation way is a, so called, brute force algorithm, in which the domain spanned by the three parameters is sampled and every possible combination is tested. The potential downside is missing optimal values since only a discrete spectrum is computed. To overcome this issue, the domain for the parameters can be determined iteratively, with e.g. starting from a very coarse grid resolution, but spanning a broad range of $\min / \max$ value for each parameter and subsequently lowering the step size as well as the min/max range to increase the tested variable pairs. Luckily, because of the characteristics of the phenological model, this simple brute force approach worked quite well. The domains for each respective variable were chosen to be: (i) $t_{0}$ : Lower bound: 1 . Upper bound: long-term mean of the respective phenological phase. (ii) $T_{b}$ : Lower bound: $0^{\circ} \mathrm{C}$. Upper bound: long-term average daily temperature of the long-term mean entry date in degree Celsius. (iii) $F^{*}$ : Lower bound: $10^{2 \circ} \mathrm{C}$. Upper bound: $10^{4 \circ} \mathrm{C}$ to $10^{5 \circ} \mathrm{C}$, depending on the phenological phase. $F^{*}$ has a more abstract characteristic. For this parameter, simply starting very low (plant development time spanning a couple of days) and iteratively working towards the optimum as described above - worked best. The parameter phase space for Aesculus hippocastanum - leaf unfolding can be seen in figure 5. The valley like range of comparable $S S R$ values is apparent and hence, guarantees the robustness of the optimisation.

For the simulated annealing approach, temperature values ranging from 0.2 to 20 and iterations from 50 to 200 were tested. The step range for the 
algorithm was randomly drawn from an uniform distribution with bounds $[-5,5]$ for $t_{0},[-3,3]$ for $T_{b}$ and [-2000,2000] for $F^{*}$. The optimal parameter values derived from the brute force approach were chosen as initial conditions. The results did not improve the optimised set of parameters derived from the brute force approach, which may be again due to the phase space characteristic and hence, the former result is used for further the application.

\subsection{Model validation}

The optimal set of parameters for each phase are shown in table 4 . Note that all except 2 phases exhibit a $F^{*}$ value of 18500 . With a step size of 500 , this suggests an optimal value between 18250 and 18750. Interestingly, the other parameters vary much more, which corresponds to $F^{*}$ boasting a quite distinct structure where the optimum is found. This can largely attributed to the time of year, where the respective phase occurs. The later the entry date, the larger $F^{*}$. Although it has to be noted, that $t_{0}$ can interact in a comparable way, compensating a lower $F^{*}$, with increasing values.

The phenological model was optimised for every E-OBS grid-point in Germany and validated against some sampled time series. Those were chosen from random grid-points and showed matching evolution in time. The three parameter values did not exhibit strong dependency on space. However, this might simply be due to the relatively small and topographically not too complex spatial extent (regional scale).

In figure 6, simulated entry-dates using the optimised parameters and the averaged E-OBS temperature data, representative for Germany, is shown. $S S R$ and the Pearson correlation coefficient are listed in each respective subplot. This pictures an in-sample test, because of course, the same data from 
which the parameters have been derived is used here to drive the model. But nevertheless, it serves as an example how well the entry date of different phenological phases can be modeled with only three degrees of freedom and furthermore, no extrapolation using a completely differing input statistic is sought after (were robust results would not be guaranteed). The only phase for which the model performed noticeable weaker than the rest, is Sambucus - first ripe fruits. This is due to the phase happening very late in the vegetation period - as can be seen on the ordinate - and thus incorporating many complex interactions in the development process (even though temperature is still the main driver). Additionally, the two phases Sambucus - beginning of flowering, id $=27$ and Tilia - beginning of flowering, id=55, exhibit a distinct worse $S S R$ and lower $\rho$, than all the other remaining phases, which can again be associated to the longer vegetation period. Note that, however, the starting date for the temperature summation in the model $\left(t_{0}\right)$, is larger for those later phenological phases, but this does not circumvent the intrinsic uncertainty in complex evolutionary plant processes, arising from the longer vegetation period.

\subsection{Application to GCM data}

Resulting phenological entry-date time series generating by feeding the phenological model with the GCM data are shown in figure 7 . Note that especially in the first few decades, little difference between the two shown experiments can be deduced. This changes in recent times, where the anthropogenic impact is much more apparent. There are some extreme outlier years, were the phenological observations (modeled with E-OBS data) reside 
outside the range of the GCM experiments. But this is simply due to the shadings only accounting for one standard deviation, hence roughly $68 \%$ of the full distribution. The associated simulated variance is shown in figure 8 , where error-bars indicate - again - one standard deviation. Contrary to the time series, it can be seen that the modeled variance from the GCMs is higher than that of the E-OBS realisation, picturing realistic ensemble behaviour, as what would be expected. The 'piControl' GCM experiments were fed into the phenological model as well. All of the generated entry-dates simulations were then handed into the consistency analysis, beginning with the test for the null hypothesis in the following section.

\section{Detection of a significant discrepancy between naturally and internally forced climate conditions and observations}

Modeled phenological data is - as the observed one - very noisy, due to high temperature year-to-year variations during the physiological development process. To assess the underlying low frequency influence of different forcings, the high frequency components have to be suppressed as much as possible. Therefore, the signal-to-noise ratio was increased by calculating 50year running trends. This reduces the time series data to sets of trends, each corresponding to one kind of forcing ('historical', 'historicalNat', 'piControl') or to observations. Those four different categories were then subject to the Mann-Whitney U-Test against each other (as outlined above).

Figure 9 shows the trend distributions as histogram plots with an overlying Gaussian kernel density estimator. The corresponding $U$-values with their respective $p$-values are shown in table 5. Significant values are marked therein 
with a shaded background. For these cases, the null hypotheses cannot be rejected, but has to be rejected for all other cases (no shading). For the used two-tailed test, the resulting significance level is 0.05 . The test itself is quite sensitive to the median, therefore large distances between them propagate into low $p$ and $U$ values (hence why the median is plotted for each sample as well). It is quite apparent, that trends resulting from naturally forced conditions or internal variability only, do not differ, in terms of the test statistic, for all phases. At the same time, they exhibit a significant deviation with respect to observations as well as 'historical' runs, which contain anthropogenic forcing. The former part suggest the rejection of the null hypothesis, that the observations can be explained with internal climate variability or naturally forced conditions alone, hence successfully detecting the soughtafter discrepancy. The $p$ and corresponding $U$ values between observations and 'historical' data show the significance of both originating from the same distribution. This is true for every phenological phase but one: Sambucus - beginning of flowering, which might be due to this phase happening very late in the vegetation period and thereby already discussed associated uncertainties. Phenological model parameters for this phase were the worst overall (in terms of the optimisation metric), indicating higher modeling error, which could potentially cascade down into the statistical evaluation as well. Furthermore, the distribution for the 'historical' experiments for this phase almost shows a bimodal behaviour, which might suggest modeling issues originating from the underlying GCMs. Lastly on this subject, this does not influence forthcoming analysis in any way, as the statistical test here only shows, that there is a discrepancy. Note that the median of 'historical' runs 
is outside the $1 \sigma$ range of internal climate variability, standalone indicating a distinct difference between those experiments. Using 30-year running trends instead of the 50-year window included much more noise, which was propagated into the evaluation, but detection based on the same criteria was still achieved for every phase (not shown).

\section{Attributing the observed change to anthropogenic causes}

The vital part in assessing the attribution issue is not if 'historical' GCM runs are consistent with observations, which can be readily assessed e.g. by the $U$ statistics, but to show the consistency between anthropogenically forced conditions and observations, while showing that other physically plausible causes are inconsistent. Therefore, the above defined consistency factor (equation 7) incorporates two experiments, from which the difference was assessed (e.g.'historical' and 'historicalNat' hence, anthropogenic impact is evaluated). This acts as a weighting factor which is applied to the $U$ value of the desired experiment (in the above case 'historical') together with the observations. Furthermore, the alternative physically plausible consistency between naturally forced conditions and observations has to be checked as well, hence why the $c$ value was calculated for all three variants mentioned above: $c_{H N O}, c_{N P O}$ and $c_{H P O}$. From these results, it can be deduced if (a) anthropogenic forcing is consistent with observations, (b) natural forcing is consistent with observations and (c) a combination of anthropogenic and natural forcing is consistent with observations. These consistency factors are shown in figure 10 for all phenological phases. The aforementioned threshold 
of $c=0.5$ is pictured as a thick, black, dashed line. From the full experiment sets, 100 realisations per category were randomly drawn and thereupon $c$ calculated. This was repeated $10^{4}$ times (bootstrap) from which associated confidence ellipses were determined (two standard deviations, which is roughly equivalent to the $95 \%$ confidence level). For the scatter plot itself, only a random subset of all the $10^{4}$ points is plotted, in order to not overload the figure. The plots can be interpreted as follows: For $c_{H N O}$ (blue), the scatter plot corresponds to the $U_{H N}^{*}$ values on the abscissa and to $U_{H O}^{*}$ for the ordinate. Thus, for $c_{H N O}$, the consistency between anthropogenic impact (distinctness between 'historical' and 'historicalNat') and observations can be deduced. The further the plotted values reside on the left, the greater the distinction between 'historical' and 'historicalNat' categories. Furthermore, high y values correspond to a small distinction between 'historical' and observations. What can be concluded, is that anthropogenic factors deviate significantly from naturally ones and that 'historical' experiments are consistent with observations. The difference between the former and $c_{H P O}$ (grey) is, that $U_{H P}^{*}$ is used for the abscissa location (as can be readily seen from the index convention for $c$ ). If the resulting values reside further to the right-hand side (10 out of 12 phases), the distinction between 'historical' and 'historicalNat' is greater than between 'historical' and 'piControl' driven data. Thereupon we can infer, that adding natural forcing onto internally forced climate conditions, the discrepancy with regards to 'historical' increases, even though, conversely, natural forcing is included in 'historical' experiment runs as well. In the other case (2 out of 12 phases), the exact opposite can be reasoned. When analysing the plots though, it has to be noted that both cases exhibit a 
significant overlap, thus the reasoned statements might be disregarded as not significant with respect to the overlapping area. On a more interesting note, $c_{N P O}$ (green) corresponds to $U_{N P}^{*}$ on the abscissa and $U_{N O}^{*}$ on the ordinate. The far-right located scatter plots indicate that data driven by 'historicalNat' shows no significant distinction to the one driven by 'piControl' experiment runs. Additionally, the ordinate position close to the bottom signifies significant difference between 'historicalNat' and observations (which was already assessed in the detection part, but can be seen here visually as well). Thus, it is apparent from $c_{N P O}$ (green), that natural forcing is not consistent with observations, thereby failing to reveal the cause. Both $c_{H N O}$ and $c_{H P O}$ show consistent results between associated forcings and observations. While for the latter - being a combination of anthropogenic and natural forcing - there would be no attribution to a single cause inferable, but when including the information of $c_{N P O}$, it is apparent that the anthropogenic forcing is the only underlying cause that is consistent with observed behaviour.

\section{Concluding remarks}

First of all, the resulting 12 phenological phases for Germany and the respective parameter optimisation were found to be quite homogeneous across the region of interest, suggesting a representative time series can be established without concern for this area. The simple brute-force approach showed comparable results to the probabilistic simulated annealing optimisation technique. Due to the nature of the phenological model parameter phase space, optimal values lying inside a broad valley, robustness in the 
optimisation performance is signified.

Averaged and bias corrected GCM data was found to adequately represent distinctly forced climate conditions. The resulting modeled phenological time series showed realistic realisations of possible climate evolutions, indicating that the calibrated phenological model did not inhibit possible extremes and outliers.

The definition of the consistency factor based on the Mann-Whitney-U-Test allowed the assessment which of the underlying forcing is a cause, consistent with observations. In this form, $c$ can be especially useful if data forced by a specific cause is not available in sufficient quantities, or not available at all. For the presented study, this was the case for experiments with only anthropogenic forcings. It is - together with natural forcings - however, included in 'historical' experiments, which were available at a much higher quantity. Together with 'historicalNat' experiments and the inclusion of their distinctness in the consistency factor, it was possible to extract the sole impact of anthropogenic forcing. As shown, the calculated consistency factors and $U$-statistics indicate: (i) A correct rejection of the null hypotheses $H_{0}$, that the observations can be explained by internal climate variability or naturally forced climate conditions only, for all 12 evaluated phenological phases. (ii) The attribution of observed phenological evolution to anthropogenic forcing. The strength of the attribution statements can readily be seen from the scatter plots of the different consistency factors. The presented technique allows for a fast quantitative assessment about which underlying cause of a signal is responsible for an observed development. It can easily be scaled up to include more 
distinguishable forcings, which can then be tested against each other. For example, one could derive the weighting from the distinctness of more than one $U^{*}$ values, thereby testing multiple physically plausible forcings at once (although to negate the effect of causes cancelling each other, each forcing should also always be investigated alone).

Additionally, since thereby employed procedures directly rely on temperatures on a sub-continental scale, the successful detection and attribution carried out for phenological phases implicitly implies the same for temperature. Although, possible distortions from the phenological model cannot be fully excluded.

Future work could be based on several findings contained in this study. The assessment of future phenological evolution, driven with climate change scenarios (downscaled climate projections), is perhaps the most obvious application. However, it has to be noted that the phenological model should be validated within a broader input range, as statistic of climate projections can be significantly different from current conditions. Findings regarding the phenological model might be of substantial importance for an application within the realm of ecology or food security. The used downscaling approach as well as the established optimisation procedure required in setting up the phenological model may be directly used for that purpose. Another interesting question is whether achieved results still hold in other as well as topographically more complex regions, too. Apart from that, it is of course worth investigating the possibility of carrying out the process of detection and attribution further, to an even higher degree of detail. This requires analyses of a wider scope of forcing factors and can possibly be done on the basis of 
the new generation of CMIP6 GCM models. Furthermore, extreme event attribution techniques can be applied to years, in which the corresponding entry date exhibited extreme outlier characteristic. Lastly, the consistency factor can be applied in other fields as well, as long as the requirements for the Mann-Whitney U Test hold.

\section{Acknowledgments}

We acknowledge the E-OBS data set from the EU-FP6 project ENSEMBLES (http://ensembles-eu.metoffice.com) and the data providers in the ECA\&D project (http:// www.ecad.eu), as well as DATAPHEN - ACRP963777 (Austrian Climate Research Programme).

We thank the Central Institute for Meteorology and Geodynamics (ZAMG) for providing a workplace for SL.

\section{Conflict of interest}

The authors declare no conflict of interest.

\section{References}

Abatzoglou, J. T., \& Brown, T. J. (2012). A comparison of statistical downscaling methods suited for wildfire applications. International Journal of Climatology, 32, 772-780.

Ahas, R., Aasa, A., Menzel, A., Fedotova, V., \& Scheifinger, H. (2002). Changes in european spring phenology. International Journal of Climatology: A Journal of the Royal Meteorological Society, 22, 1727-1738. 
Bentsen, M., Bethke, I., Debernard, J., Iversen, T., Kirkevåg, A., Seland, Ø., Drange, H., Roelandt, C., Seierstad, I., Hoose, C. et al. (2013). The norwegian earth system model, noresm1-m - part 1: Description and basic evaluation of the physical climate. Geosci. Model Dev, 6, 687-720.

Van den Besselaar, E., Haylock, M., Van der Schrier, G., \& Klein Tank, A. (2011). A european daily high-resolution observational gridded data set of sea level pressure. Journal of Geophysical Research: Atmospheres, 116.

Chuine, I., Cambon, G., \& Comtois, P. (2000). Scaling phenology from the local to the regional level: advances from species-specific phenological models. Global Change Biology, 6, 943-952.

Chuine, I., Cour, P., \& Rousseau, D. (1998). Fitting models predicting dates of flowering of temperate-zone trees using simulated annealing. Plant, Cell E Environment, 21, 455-466.

Chuine, I., Cour, P., \& Rousseau, D. (1999). Selecting models to predict the timing of flowering of temperate trees: implications for tree phenology modelling. Plant, Cell \&5 Environment, 22, 1-13.

Chuine, I., Kramer, K., \& Hänninen, H. (2003). Plant development models, phenology: an integrative environmental science. Kluwer, the Netherlands. Corlett, RT $\& 3$ Lafrankie, JV (1998) Potential impacts of climate change on tropical Asian forests through an influence on phenology. Climatic Change, 39, 439-453.

Dean, S., \& Stott, P. (2009). The effect of local circulation variability on the 
detection and attribution of new zealand temperature trends. Journal of Climate, 22, 6217-6229.

Haylock, M., Hofstra, N., Klein Tank, A., Klok, E., Jones, P., \& New, M. (2008). A european daily high-resolution gridded data set of surface temperature and precipitation for 1950-2006. Journal of Geophysical Research: Atmospheres, 113.

Hegerl, G., \& Zwiers, F. (2011). Use of models in detection and attribution of climate change. Wiley interdisciplinary reviews: climate change, 2, $570-591$.

Hegerl, G. C., Hasselmann, K., Cubasch, U., Mitchell, J. F., Roeckner, E., Voss, R., \& Waszkewitz, J. (1997). Multi-fingerprint detection and attribution analysis of greenhouse gas, greenhouse gas-plus-aerosol and solar forced climate change. Climate Dynamics, 13, 613-634.

Hegerl, G. C., Hoegh-Guldberg, O., Casassa, G., Hoerling, M. P., Kovats, R., Parmesan, C., Pierce, D. W., Stott, P. A. et al. (2010). Good practice guidance paper on detection and attribution related to anthropogenic climate change. In Meeting Report of the Intergovernmental Panel on Climate Change Expert Meeting on Detection and Attribution of Anthropogenic Climate Change. IPCC Working Group I Technical Support Unit, University of Bern.

Hofstra, N., Haylock, M., New, M., Jones, P., \& Frei, C. (2008). Comparison of six methods for the interpolation of daily, european climate data. Journal of Geophysical Research: Atmospheres, 113. 
Hunter, A. F., \& Lechowicz, M. J. (1992). Predicting the timing of budburst in temperate trees. Journal of Applied Ecology, (pp. 597-604).

IPCC-AR5 (2014). Climate change 2013: the physical science basis: Working Group I contribution to the Fifth assessment report of the Intergovernmental Panel on Climate Change /Stocker, T.F., D. Qin, G.-K. Plattner, M. Tignor, S.K. Allen, J. Boschung, A. Nauels, Y. Xia, V. Bex and P.M. Midgley (eds.)]. Cambridge University Press. doi:10.1017/ CB09781107415324.

Karoly, D. J., Braganza, K., Stott, P. A., Arblaster, J. M., Meehl, G. A., Broccoli, A. J., \& Dixon, K. W. (2003). Detection of a human influence on north american climate. Science, 302, 1200-1203.

Mann, H. B., \& Whitney, D. R. (1947). On a test of whether one of two random variables is stochastically larger than the other. The annals of mathematical statistics, (pp. 50-60).

Maurer, E. P. (2010). The utility of daily large-scale climate data in the assessment of climate change impacts on daily streamflow in california. Hydrology and Earth System Sciences, .

Maurer, E. P., Das, T., \& Cayan, D. R. (2013). Errors in climate model daily precipitation and temperature output: time invariance and implications for bias correction. Hydrology and Earth System Sciences, .

Meier, U. (2001). Bbch-monograph: growth stages of mono-and dicotyledonous plants. Federal Biological Research Centre for Agriculture and Forestry, (pp. 130-133). 
Menzel, A., Sparks, T. H., Estrella, N., Koch, E., Aasa, A., Ahas, R., AlmKübler, K., Bissolli, P., Braslavská, O., Briede, A. et al. (2006). European phenological response to climate change matches the warming pattern. Global change biology, 12, 1969-1976.

Metropolis, N., Rosenbluth, A. W., Rosenbluth, M. N., Teller, A. H., \& Teller, E. (1953). Equation of state calculations by fast computing machines. The journal of chemical physics, 21, 1087-1092.

Panofsky, H. A., Brier, G. W., \& Best, W. H. (1958). Some application of statistics to meteorology. Earth and Mineral Sciences Continuing Education, (p. 224).

Root, T. L., Price, J. T., Hall, K. R., Schneider, S. H. et al. (2003). Fingerprints of global warming on wild animals and plants. Nature, 421, 57.

Rosenzweig, C., Karoly, D., Vicarelli, M., Neofotis, P., Wu, Q., Casassa, G., Menzel, A., Root, T. L., Estrella, N., Seguin, B. et al. (2008). Attributing physical and biological impacts to anthropogenic climate change. Nature, 453, 353.

Rosenzweig, C., \& Neofotis, P. (2013). Detection and attribution of anthropogenic climate change impacts. Wiley interdisciplinary reviews: climate change, 4, 121-150.

Scheifinger, H., Menzel, A., Koch, E., \& Peter, C. (2003). Trends of spring time frost events and phenological dates in central europe. Theoretical and Applied Climatology, 74, 41-51. 
Schönwiese, C.-D. (2013). Praktische Statistik für Meteorologen und Geowissenschaftler. Schweizerbart'sche Verlagsbuchhandlung.

Stone, D. A., Allen, M. R., Stott, P. A., Pall, P., Min, S.-K., Nozawa, T., \& Yukimoto, S. (2009). The detection and attribution of human influence on climate. Annual Review of Environment and Resources, 34.

Stott, P. A., Christidis, N., Otto, F. E., Sun, Y., Vanderlinden, J.-P., Van Oldenborgh, G. J., Vautard, R., Von Storch, H., Walton, P., Yiou, P. et al. (2016). Attribution of extreme weather and climate-related events. Wiley Interdisciplinary Reviews: Climate Change, 7, 23-41.

Stott, P. A., Tett, S., Jones, G., Allen, M., Mitchell, J., \& Jenkins, G. (2000). External control of 20th century temperature by natural and anthropogenic forcings. science, 290, 2133-2137.

Taylor, K. E., Stouffer, R. J., \& Meehl, G. A. (2012). An overview of cmip5 and the experiment design. Bulletin of the American Meteorological Society, 93, 485-498.

Templ, B., Koch, E., Bolmgren, K., Ungersböck, M., Paul, A., Scheifinger, H., Busto, M., Chmielewski, F.-M., Hájková, L., Hodzić, S. et al. (2018). Pan european phenological database (pep725): a single point of access for european data. International journal of biometeorology, 62, 1109-1113.

Tett, S. F., Stott, P. A., Allen, M. R., Ingram, W. J., \& Mitchell, J. F. (1999). Causes of twentieth-century temperature change near the earth's surface. Nature, 399, 569. 
Thrasher, B., Maurer, E. P., Duffy, P. B., \& McKellar, C. (2012). Bias correcting climate model simulated daily temperature extremes with quantile mapping. Hydrology and Earth Systems Science, 16.

Voldoire, A., Sanchez-Gomez, E., y Mélia, D. S., Decharme, B., Cassou, C., Sénési, S., Valcke, S., Beau, I., Alias, A., Chevallier, M. et al. (2013). The cnrm-cm5. 1 global climate model: description and basic evaluation. Climate Dynamics, 40, 2091-2121.

Von Storch, H., Zorita, E., \& Cubasch, U. (1993). Downscaling of global climate change estimates to regional scales: an application to iberian rainfall in wintertime. Journal of Climate, 6, 1161-1171.

Walther, G.-R., Post, E., Convey, P., Menzel, A., Parmesan, C., Beebee, T. J., Fromentin, J.-M., Hoegh-Guldberg, O., \& Bairlein, F. (2002). Ecological responses to recent climate change. Nature, 416, 389-395.

Wilcoxon, F. (1947). Probability tables for individual comparisons by ranking methods. Biometrics, 3, 119-122.

Wilcoxon, F. (1992). Individual comparisons by ranking methods. In Breakthroughs in statistics (pp. 196-202). Springer. 
Images (Figures, Tables)

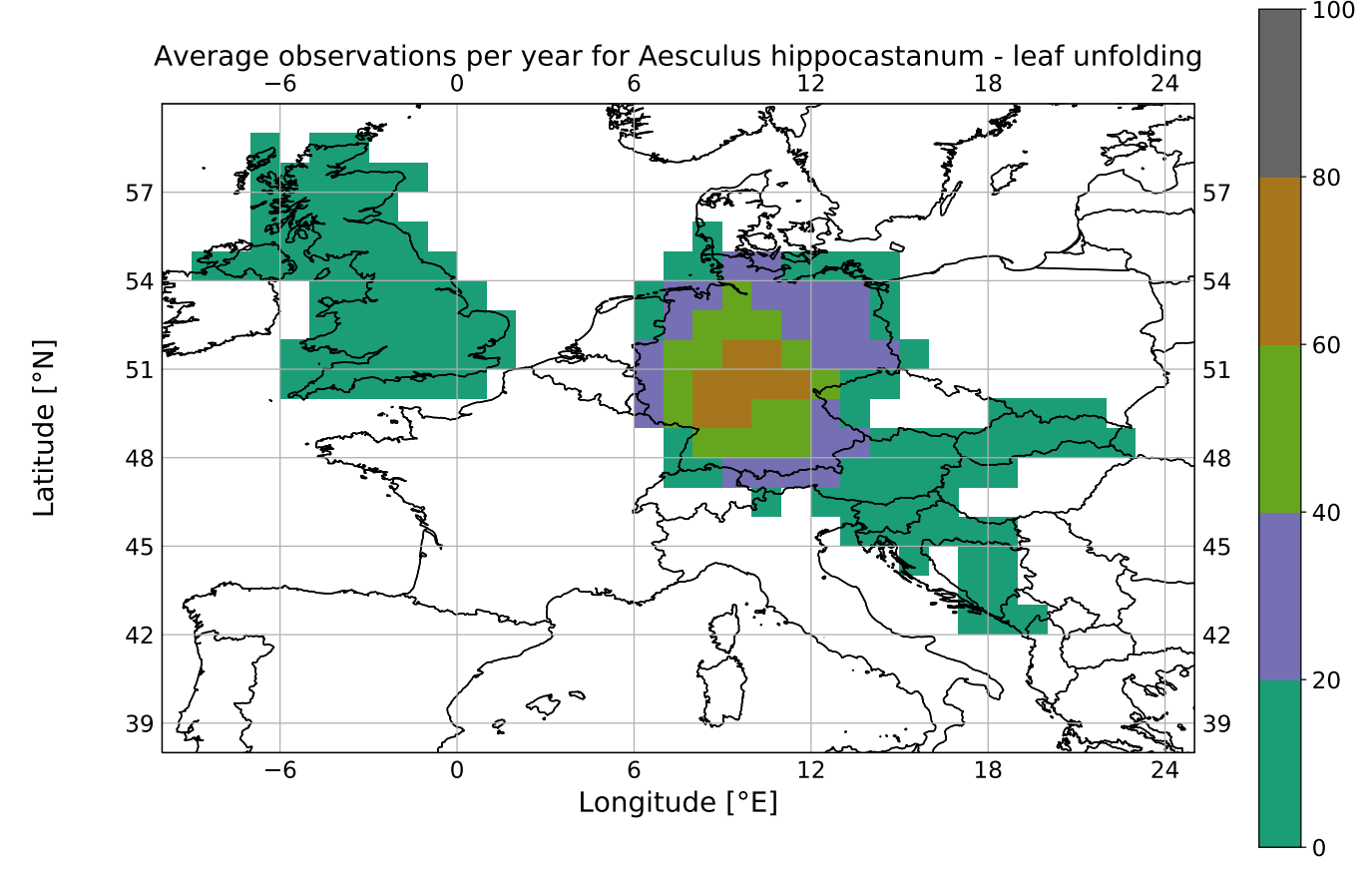

Figure 1: Average station observations per year for the phenological phase Aesculus hippocastanum - leaf unfolding. Note the density differences between countries. In central Europe, Germany stands out with the most dense observational record. 


\begin{tabular}{lllll}
\hline Pheno index & Pheno ID & \multicolumn{1}{c}{ Scientifc name } & \multicolumn{1}{c}{ Species name } & Phase \\
\hline 1 & 101011 & Aesculus hippocastanum & Horse chestnut & leaf unfolding \\
2 & 101060 & Aesculus hippocastanum & Horse chestnut & beginning of flowering \\
11 & 106011 & Betula & Birch & leaf unfolding \\
15 & 108011 & Fagus & Beech & leaf unfolding \\
\hline 21 & 110010 & Picea abies (P.excelsa) & Spruce & first leaves separated \\
\hline 23 & 111011 & Quercus robur (Q.peduncula) & Pedunculate oak & leaf unfolding \\
\hline 27 & 112060 & Sambucus & Elder & beginning of flowering \\
\hline 29 & 112086 & Sambucus & Elder & first ripe fruits \\
52 & 127060 & Syringa vulgaris & Lilac & beginning of flowering \\
\hline 53 & 128060 & Taraxacum officinale & Common dandelion & beginning of flowering \\
\hline 55 & 129060 & Tilia & Linden & beginning of flowering \\
\hline 82 & 223060 & Prunus cerasus & Sour cherry & beginning of flowering \\
\hline
\end{tabular}

Table 1: The twelve phenological phases considered for further investigation. The first column consists of a running index inside the PEP725 data set, the second one is an identification number, for which the first three digits correspond to the plant species and the last three to the phenological phase (see Meier (2001)). 
Yearday distribution 1951 - 1970 (blue) vs 1996 - 2015 (red), dashed line: $\mu$

Aesculus hippocastanum

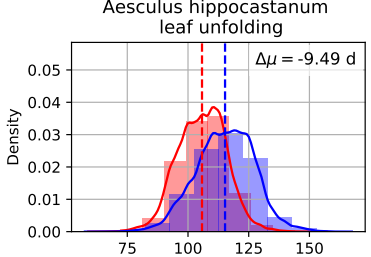

Picea abies (P.excelsa)

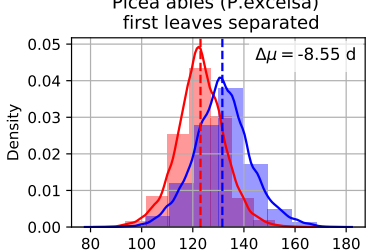

Syringa vulgaris

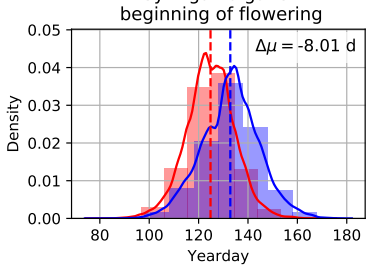

Aesculus hippocastanum beginning of flowering
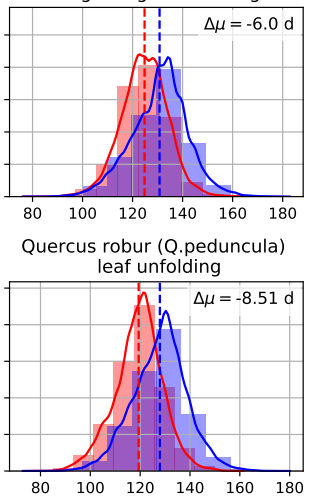

Taraxacum officinale

beginning of flowering

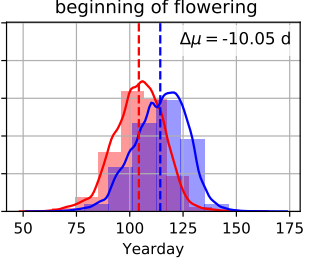

Betula
leaf unfolding

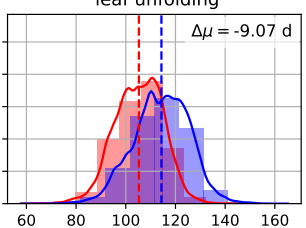

Sambucus

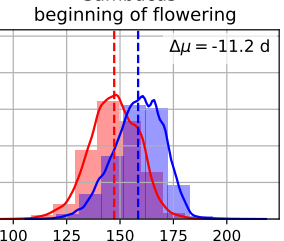

150

beginning of flowering



Fagus
leaf unfolding

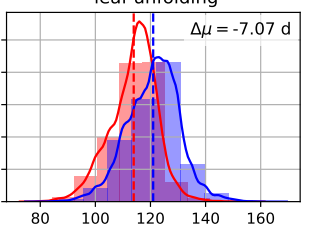

Sambucus first ripe fruits

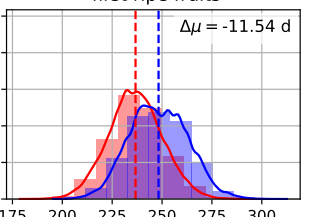

$\begin{array}{llllll}175 & 200 & 225 & 250 & 275 & 300\end{array}$

Prunus cerasus

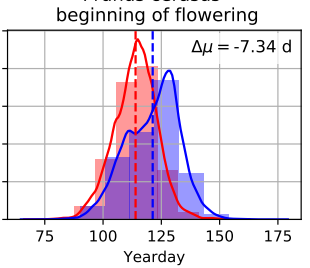

Figure 2: Change in the distribution of phenological observations between the periods 1951-1970 and 1996-2015 (spatially averaged in Germany per year). 


\begin{tabular}{cccccc}
\hline Model & piControl & historical & historicalExt historicalNat & spatial resolution in [ ${ }^{\circ}$ ] lat x lon \\
\hline CanESM2 $^{1}$ & 1095 & 4 & 4 & 5 & $2.8 \times 2.8$ \\
CCSM4 $^{2}$ & 155 & - & - & 4 & $0.9 \times 1.25$ \\
CNRM-CM5 $^{1}$ & 850 & 7 & 7 & 6 & $1.4 \times 1.4$ \\
GFDL-CM3 $^{3}$ & 800 & 1 & - & 3 & $2.0 \times 2.5$ \\
IPSL-CM5A-LR $^{2}$ & 1000 & 4 & - & 3 & $1.9 \times 3.75$ \\
NorESM1-M & 500 & 3 & 3 & 1 & $1.9 \times 2.5$ \\
\hline Total & 4400 & 19 & 14 & 22 & \\
\hline${ }^{1} 18500101-20121231$, & ${ }^{2} 18500101-20051231$, & ${ }^{3} 18600101-20051231$ &
\end{tabular}

Table 2: Overview of included GCMs. The numbers in the second column 'piControl' correspond to the amount of years available, the others (columns three to five) to the quantity of ensemble members. 




Figure 3: Quantiles of entry dates against altitude (left), latitude (middle) and longitude (right), calculated in bins of $200 \mathrm{~m}, 1^{\circ}$ and $1^{\circ}$ steps respectively, for Aesculus hippocastanum - leaf unfolding. The shown numbers correspond to the quantity of observations in each bin. Lines go from the 5 th percentile (bottom), in $5 \%$ steps up to the 95 th percentile. 


\begin{tabular}{lllllllllllll}
\hline Pheno index & 1 & 2 & 11 & 15 & 21 & 23 & 27 & 29 & 52 & 53 & 55 & 82 \\
\hline Coeff. & 0.0131 & 0.0126 & 0.0105 & 0.0075 & 0.012 & 0.0117 & 0.0135 & 0.0121 & 0.0122 & 0.013 & 0.0172 & 0.0101 \\
\hline
\end{tabular}

Table 3: Altitude regression coefficients for phenological entry dates from the MLR model. Only data under $1000 \mathrm{~m}$ was used, as there were too few observations at higher altitudes to guarantee statistical robustness. 
RMSE mean near-surface temperature (1951-2005) for GCM Ensembles, reference: E-OBS Uncorrected (dashed), \& corrected (solid)



Figure 4: Root Mean Square Error (RMSE) for mean near-surface temperature per yearday (over the full time period) for 'historical' experiments (blue) and 'historicalNat' experiments (green) with respect to E-OBS data. Raw GCMs as dashed, corrected ones as solid lines. 


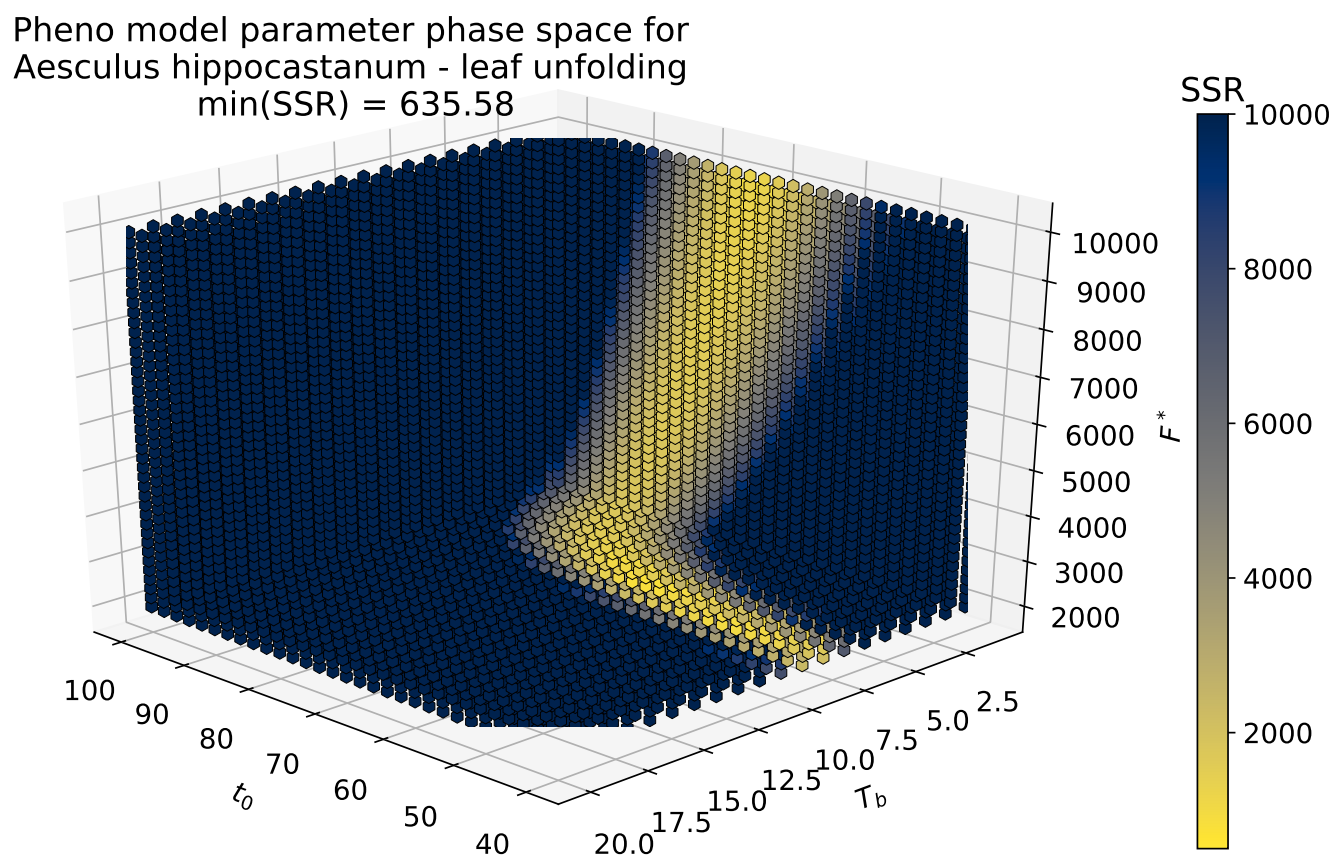

Figure 5: Phenology model parameter phase space for the three parameters to be optimized for Aesculus hippocastanum - leaf unfolding. The coloring corresponds to $S S R$ (summed squared residuals) values, hence lower values represent higher agreement between the model and observations. 


\begin{tabular}{ccccc}
\hline Pheno index & $t_{0}[\mathrm{~d}]$ & $T_{b}\left[{ }^{\circ} \mathrm{C}\right]$ & $F^{*}\left[{ }^{\circ} \mathrm{C}\right]$ & $S S R$ \\
\hline 1 & 26 & 1 & 16000 & 525 \\
2 & 11 & 5 & 18500 & 377 \\
11 & 16 & 1 & 18500 & 307 \\
15 & 36 & 1 & 18500 & 371 \\
21 & 26 & 4 & 18500 & 464 \\
23 & 31 & 3 & 18500 & 342 \\
27 & 61 & 7 & 18500 & 1299 \\
29 & 117 & 6 & 86000 & 2275 \\
52 & 16 & 5 & 18500 & 572 \\
53 & 1 & 2 & 18500 & 696 \\
55 & 91 & 9 & 18500 & 906 \\
82 & 26 & 2 & 18500 & 558 \\
\hline
\end{tabular}

Table 4: Optimal set of phenological model parameters for the twelve phenological phases. 
Observed pheno time series (red) vs modelled (E-OBS data, dashed black)
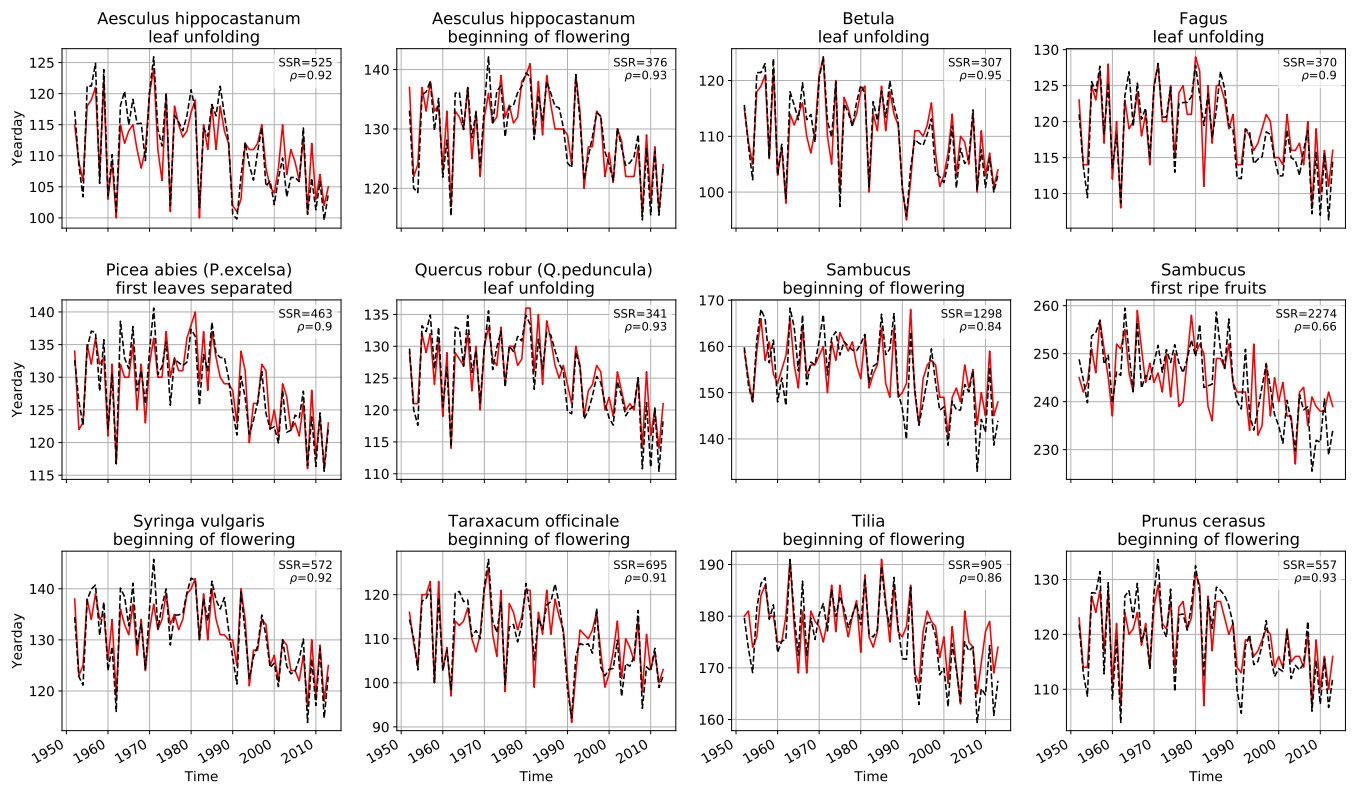

Figure 6: Phenological time series model validation: Observations (red) and modelled E-OBS data (dashed black) averaged over Germany. SSR: summed squared residuals between observations and model; $\rho$ : pearson correlation coefficient. 

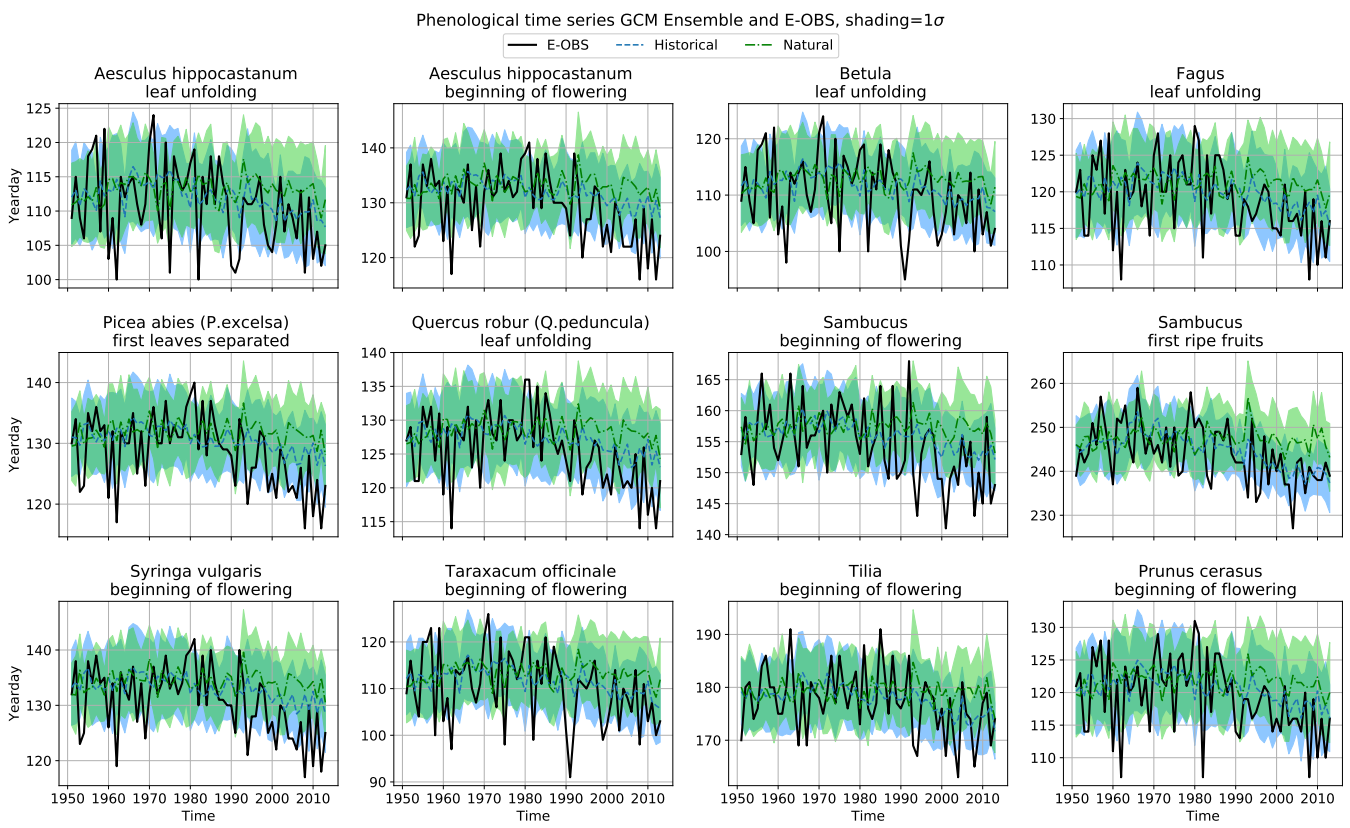

Figure 7: Modelled phenological entry-date time series for 'historical' GCMs (blue), 'historicalNat' (green) - both bias corrected - and E-OBS (black) data. Shading indicates one standard deviation. 


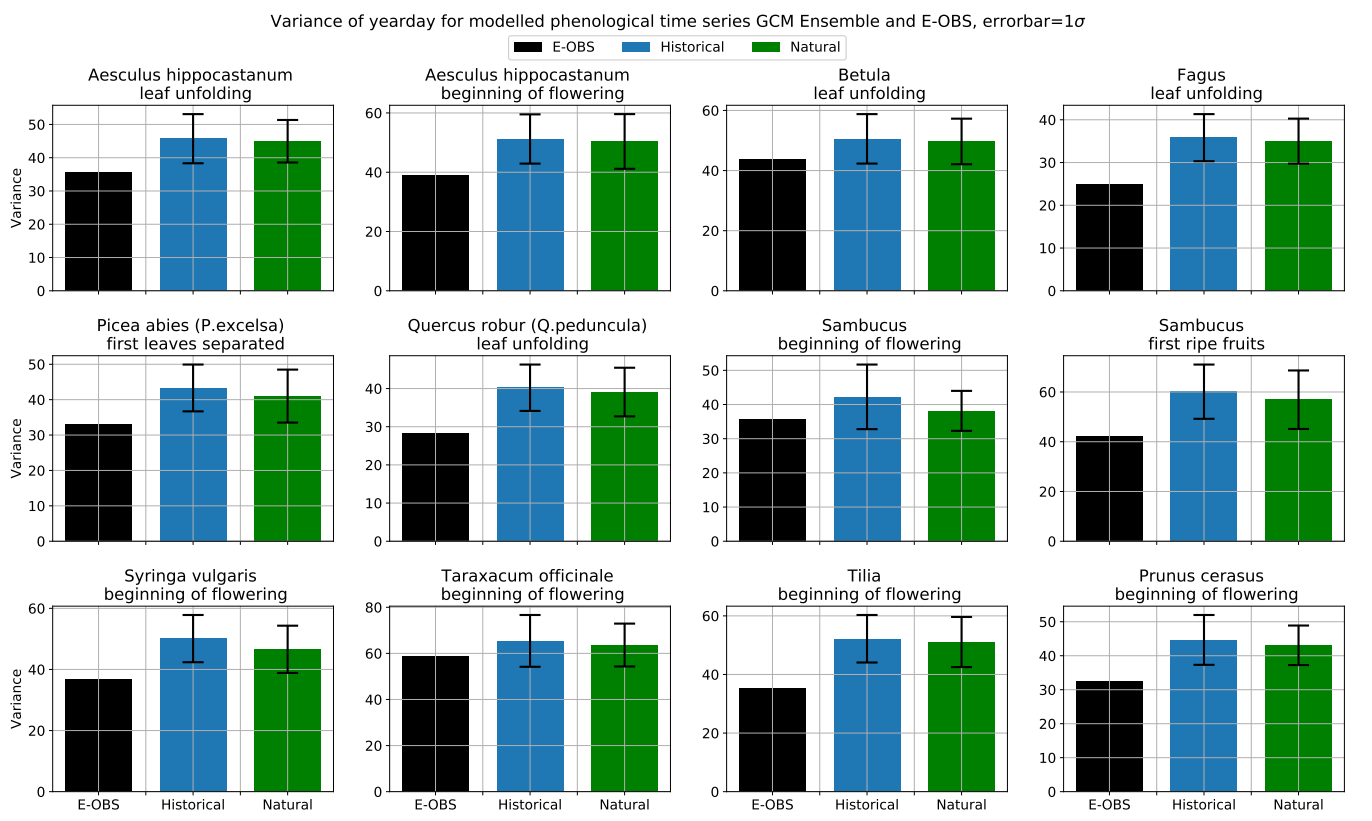

Figure 8: Variance of modelled entry dates for data as in figure 7. Errorbars indicate one standard deviation. 


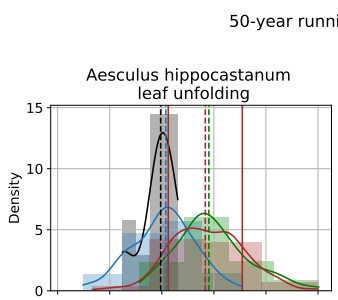

Picea abies (P.excelsa)

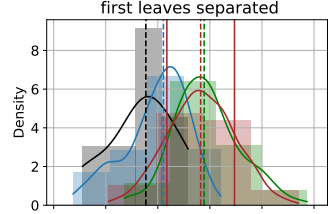

Syringa vulgaris



trends (1951-2012) GCM Ensemble and E-OBS, dashed-lines=median, brown-solid-lines $=1 \sigma$ — E-OBS $n=13$ - Historical $n=205$ - Natural $n=223$ - Internal $n=858$

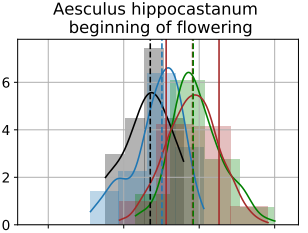

Quercus robur (Q.peduncula)

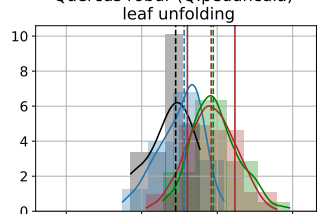

Taraxacum officinale

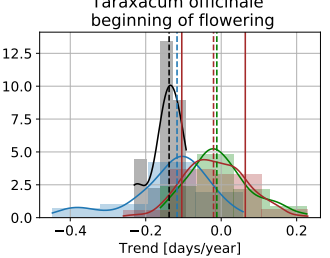

Betula
leaf unfolding

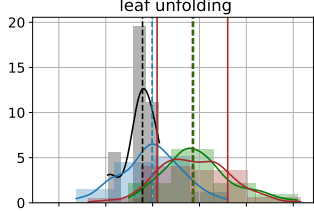

Sambucus

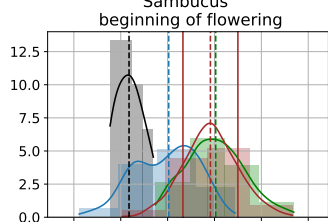

Tilia

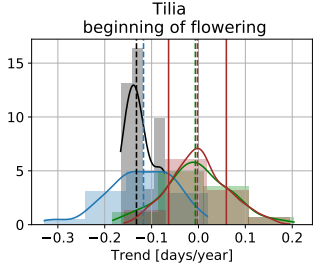

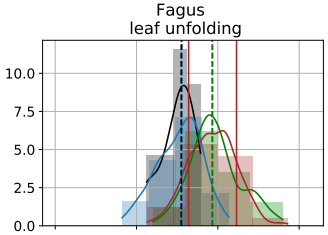

Sambucus

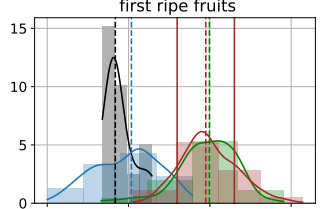

Prunus cerasus

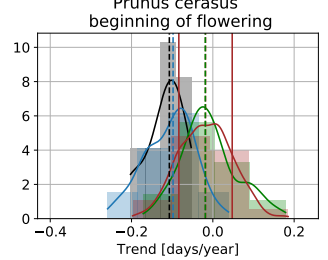

Figure 9: 50-year running trend distributions for E-OBS (black), 'historical' (blue), 'historicalNat' (green) and 'piControl' experiments (brown). Associated statistical analysis of the Mann-Whitney U-Test is shown in table 5. Dashed lines indicate median values of their respective distribution, solid brown vertical lines one standard deviation for internal climate variability ('piControl' experiments). 




Table 5: Statistical analysis for testing the null hypothesis, that two samples originate from the same basic population. Shading indicates significant outcome, where the null hypothesis cannot be rejected. No shading suggests the contrary, leading to the rejection of the null hypothesis and thereby detection of a significant discrepancy. 


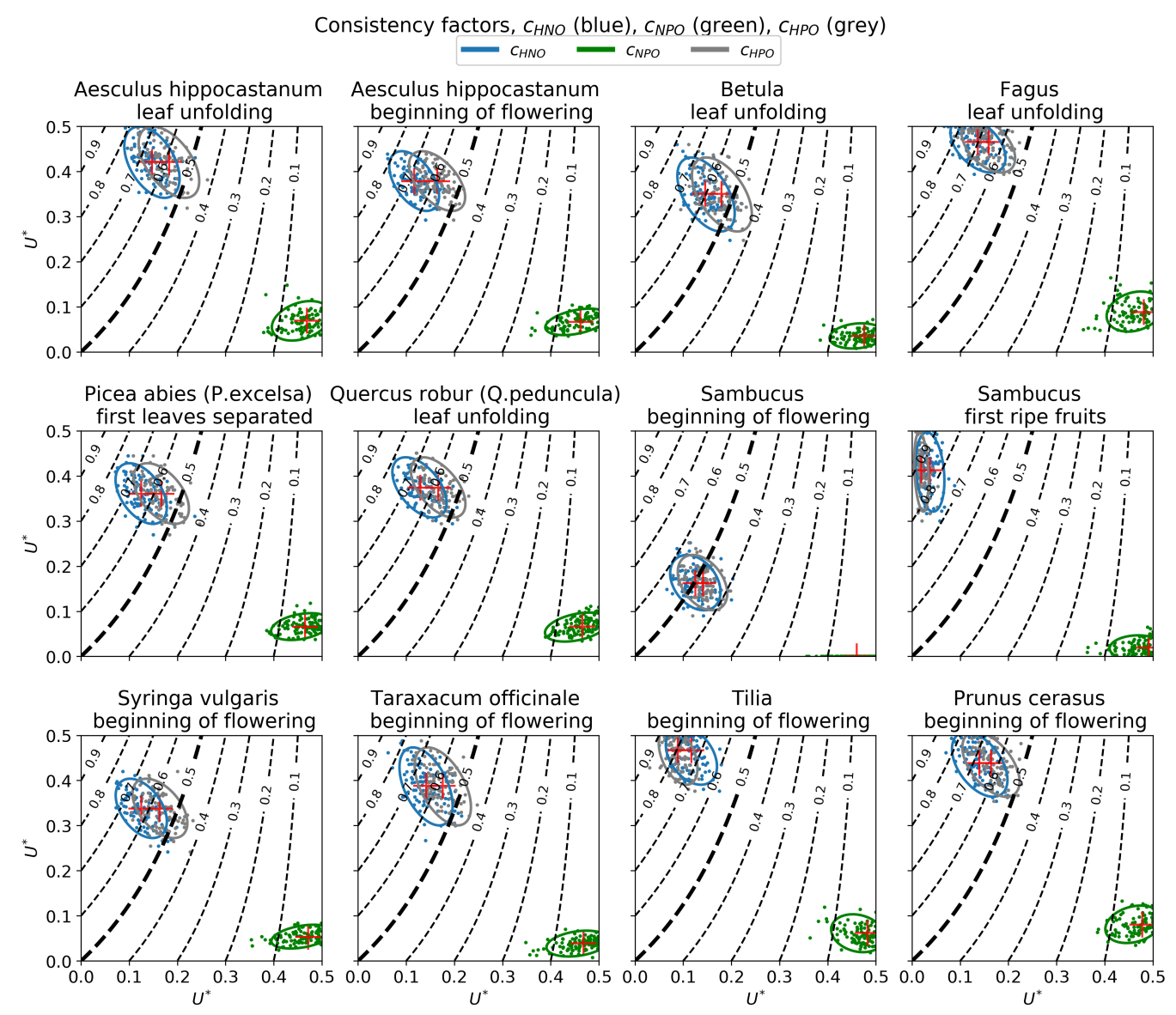

Figure 10: Consistency factors $c_{H N O}, c_{N P O}$ and $c_{H P O} . U^{*}$ on the abscissa corresponds to the respective $x, y$ samples indicated by the index convention $c_{x y z}$ and on the ordinate to $x, z$. Ellipses feature two standard deviations (roughly $95 \%$ confidence level). 